Síntesis:

Con el propósito de establecer el conocimiento acerca del género que están adquiriendo los niños y las niñas a través de la televisión, se invitó a 4 niños y 4 niñas de segundo grado de primaria a un talle en el que proporcionaron información sobre sus hábitos de consumo televisivo y sobre las características de sus programas y personajes preferidos. Adicionalmente, los participantes examinaron el contenido

de sus programas preferidos, teniendo en cuenta como categorías para el análisis del género: a) las actividades que se representan en los mensajes y que ilustran la división sexual del trabajo en términos de roles productivos, reproductivos y de participación comunitaria; b) las características emocionales que los participantes perciben de los personajes del programa elegido; c) las relaciones de poder que se representan y que se expresan a través de la posición que ocupan los personajes en la trama y la manera como resuelven los conflictos, toman decisiones y participan en las actividades. Se encontró que las interpretaciones de género que realizaron los niños y las niñas de sus programas preferidos dan cuenta de fuertes estereotipos de género y actitudes sexistas. Su apreciación de los personajes de sus programas preferidos evidencia que en las producciones televisivas persiste la elección de roles femeninos y masculinos fuertemente estereotipados, opuestos y complementarios, lo cual les impide acceder a modelos de identificación diversos en los que la masculinidad no esté asociada, exclusivamente, con la violencia y el ejercicio del poder y la feminidad con la belleza física, la expresión emocional y las relaciones sociales. Palabras clave: televisión, género, infancia, socialización.

Synthesis: In order to establish the knowledge about the genre that boys and girls are acquiring through television, 4 boys and 4 girls of second grade were invited to a workshop in which they provided information on their habits of television consumption and about the characteristics of their programs and preferred characters. Additionally, the participants examined the contents of their preferred programs, having in account like categories for the analysis of the genre: a ) the activities that are represented in the messages and that illustrate the sexual division of work in terms of productive, reproductive roles and of community participation; b) the emotional characteristics that participants perceive in the characters of the elected program; $c$ ) the power relations that are represented and that are expressed through the position that the characters have in the plot and the way that they solve conflicts,

take decisions and participate in activities. It was found that the genre interpretations realized by boys and girls of their preferred programs gives strong stereotypes of genre and sexist attitudes. Their appreciation of the characters from their preferred programs evidence that in their television productions persists the choice of feminine and masculine roles strongly stereotyped, opposite and complementary,

which makes it impossible to access diverse identification models

in which masculinity is not associated exclusively with violence, and

the exercise of power and femininity with the physical beauty, the emotional expression and social relationships. Key Words: television, gender, childhood socialization.

\section{Televisión y género: un análisis desde la perspectiva de los niños y las niñas ${ }^{1}$}

\author{
Elvia Vargas Trujillo, Ph.D \\ Facultad de Ciencias Sociales, Universidad de los Andes \\ e-mail: elvargas@uniandes.edu.co \\ Ángela María Rojas Martínez, M.Ps. \\ Facultad de Ciencias de la Comunicación \\ Corporación Universitaria Minuto de Dios \\ e-mail: amrojas@uniminuto.edu \\ Paola Balanta, M.Ps. \\ Facultad de Ciencias Sociales, \\ Universidad de los Andes \\ e-mail: p-balant@uniandes.edu.co \\ Fecha de recepción: 07-07-08 \\ Fecha de aprobación: 15-08-2008
}

Hoysereconocequelosmedios decomunicación, particularmente la televisión, ocupan un lugar cada vez más importante como agentes de socialización (Charles, 2002). En la infancia "la televisión representa una ventana hacia el mundo"2, permite a los niños y las niñas acceder a información

${ }^{1}$ Este artículo describe los resultados de la primera fase de la investigación titulada: Una propuesta dirigida al público infantil para ver televisión con perspectiva de género. El estudio completo pretende diseñar y evaluar una propuesta de formación dirigida a niños y niñas de 7 a 10 años de edad residentes en el municipio de Soacha (Colombia) con el propósito de contribuir a la construcción de relaciones de género equitativas en el grupo de pares, mediante el fomento de habilidades críticas hacia aquellos mensajes televisivos que promueven y refuerzan normas y expectativas de género inequitativas en la teleaudiencia infantil.

${ }^{2}$ AUFENANGER, S, "Stimuli, not set answers", en: Televizion 18, 2005, pág. 53. 
sobre diversas culturas, al mismo tiempo que observan comportamientos, normas, formas de relación, ideas, tradiciones y costumbres características de su contexto social.

Coherentemente con este planteamiento, la evidencia disponible señala que a través de la televisión los niños y las niñas están expuestos y expuestas a múltiples mensajes referentes a lo que significa ser hombre y ser mujer y que dichos mensajes influyen significativamente en la noción que construyen de sí mismos y en la manera como entienden, interpretan y actúan en el mundo (Götz, Hofmann, Brosius, et. al., 2008; Hains, 2008; Thompson \& Zerbinos, 1997, Vargas Trujillo, Barrera, Burgos \& Daza, 2004, entre otros).

Por lo anterior, en este estudio se buscó conocer las interpretaciones con relación al género que hacen los niños y las niñas de los mensajes que observan en sus programas preferidos. El género incluye el conjunto de atributos y comportamientos que socialmente se definen como propios de uno u otro sexo, al igual que las normas que regulan tanto las expresiones de la masculinidad y la feminidad como las relaciones entre los hombres y las mujeres (Caséz, 1998).

Contrariamente a lo que ha sido habitual en la investigación sobre el tema de género en la televisión, en este trabajo el análisis no se centró en la identificación, por parte de los adultos, de las diferencias y de los tipos de discriminación a la que están expuestos los niños y las niñas a través de los mensajes que observan.

Para los propósitos de esta investigación se involucró a las niñas y a los niños en el proceso de indagación sobre el contenido de sus programas preferidos, teniendo en cuenta como categorías para el análisis del género: a) las actividades que se representan en los mensajes y que ilustran la división sexual del trabajo en términos de roles productivos, reproductivos y de participación comunitaria; b) las características, intereses y potencialidades de los distintos implicados en los mensajes televisivos; c) las relaciones de poder que se establecen entre los personajes y que se expresan a través de la posición que ocupan en la trama y la manera como resuelven los conflictos, toman decisiones y participan en las actividades.

La relevancia del estudio que se sintetiza en este informe se fundamenta en los hallazgos investigativos que indican que, aunque la mayoría de la población mundial se enfrenta diariamente a mensajes provenientes de múltiples medios de comunicación masiva, existen unos grupos de población más vulnerables a su influencia que otros. A partir de los datos disponibles, asumimos que el impacto de la televisión es mayor en la infancia y la juventud, particularmente en el caso de los estratos bajos, debido a que este medio de comunicación constituye su principal fuente de entretenimiento, relación con el mundo y posibilidad de aprendizaje (Niño, 2004; Rincón, 2004). Adicionalmente, porque los programas televisivos que más prefieren son novelas y seriados (Niño, 2004), justamente los que se caracterizan por altos niveles de contenido sexista (Fox y Santos, 2002).

Evidentemente, entre la transmisión de los mensajes televisivos y sus efectos en las audiencias median diversos factores. Los padres pueden modular el efecto de los contenidos mediante la comunicación, esto es, acompañando a sus hijos mientras ven televisión y propiciando un ambiente en el que se pueda hablar acerca del contenido de los programas (Strasburger, 1995). Otros estudios señalan que cuando los adultos supervisan el uso de la televisión y les ofrecen la oportunidad de practicar otras actividades en su tiempo libre inciden en la forma como hijos e hijas responden a la televisión (Verma \& Larson, 2002). 
También hay hallazgos que sugieren que no es la cantidad de televisión que se ve lo que más influye sobre las actitudes, expectativas y comportamientos del televidente, sino la relación que éste tiene con el contenido del programa; su motivación para ver televisión, la forma como selecciona los programas, el grado de realismo que le atribuye a la trama y la identificación que establece con los protagonistas (ver por ejemplo, Vargas Trujillo, Barrera, Burgos y Daza, 2003; Ward, 2002; Ward \& Rivadeneyra, 1999).

Otros investigadores, por su parte, afirman que es imposible no aprender de la televisión (Göłz, 2005), sin embargo, no todas las personas aprenden lo mismo, aunque observen programas televisivos idénticos. Esto obedece a que cada individuo presta atención a aspectos específicos de los programas, aquéllos con los cuales se puede relacionar. Luego interpreta esos aspectos utilizando tanto el conocimiento previamente adquirido como la propia experiencia $y$, finalmente, integra los elementos del programa a la comprensión del mundo que está desarrollando. La pregunta que surge a partir de estos planteamientos es iqué conocimiento acerca del género están adquiriendo los niños y las niñas a través de la televisión? Responder a este interrogante fue el propósito de este estudio.

\section{Metodología}

Tipo de estudio

Se realizó un estudio descriptivo de tipo cualitativo.

\section{Participantes}

Se invitó a participar en un taller a 4 niñas y 4 niños de 7 a 9 años de edad, estudiantes del colegio Bolívar de Soacha. El taller, que tuvo dos horas de duración, se programó durante la jornada escolar, de común acuerdo con las directivas del plantel. Los 8 niños y niñas que conformaron la muestra pertenecían a los cuatro grupos de segundo grado y se seleccionaron al azar de 33 que contaban con el consentimiento informado de sus acudientes para participar en el estudio.

\section{Procedimiento}

En el taller se realizaron tres actividades: con base en una guía de entrevista, previamente validada por un experto en investigación cualitativa y en el tema de género, primero, se exploraron los hábitos de consumo televisivo de los participantes y, luego, se les solicitó que dibujaran sus personajes favoritos de la televisión y que los describieran (cómo son, qué hacen, cómo se relacionan con otras personas). A continuación, cada participante analizó su programa preferido desde la perspectiva de género, utilizando para tal fin un formato diseñado por el equipo de investigación.

El formato para el análisis del programa desde la perspectiva de género se organizó en cuatro apartados y constaba en total de 61 ítems. El primer apartado, de 17 ítems, recogía información relacionada con el programa favorito de cada participante y los roles productivos, reproductivos y de participación de los personajes, por ejemplo: ¿quién hace las compras? ¿quién trabaja?, ¿quién hace deporte? El segundo, incluía 22 ítems y recogía información sobre las relaciones de poder que se establecen entre los personajes, por ejemplo: ¿quién decide las cosas importantes?, ¿quién decide los castigos? La tercera parte incluía 8 ítems y permitía establecer las características emocionales que los niños perciben de los personajes del programa elegido, por ejemplo, alegría, preocupación o tristeza. La última parte incluía 14 ítems y recogía información sobre las expresiones de liderazgo positivo y negativo de los personajes según la percepción de los niños y 
niñas, por ejemplo, quién es el más querido, quién es el más exitoso.

\section{Resultados}

Hábitos de consumo televisivo

En la tabla 1 se encuentran los programas preferidos por los niños y las niñas participantes en el taller. Al realizar el análisis de las características generales de los programas mencionados como favoritos por los niños y las niñas, se observa que el horario de emisión varía y se distribuye a lo largo del día y de la noche.

Adicionalmente, se encontró que la mayoría de los programas preferidos por los niños y las niñas se emiten a través de canales por suscripción destinados específicamente a la audiencia infantil durante todo el día. En la televisión por suscripción, la disponibilidad de canales infantiles permite el acceso de los niños y las niñas a una gran variedad de programas, en cualquier horario. En estos casos, la audiencia requiere mayores niveles de selectividad, si se tiene en cuenta que la mayoría de los niños y las niñas permanecen gran parte del día, e incluso de la noche, solos o en compañía de personas que no ejercen control o acompañamiento y seguimiento de los programas que ven.

\section{Ver tabla No.1 en la siguiente columna}

Con respecto a los horarios en que los niños dicen ver televisión, en la tabla 2 se aprecia que esta actividad se realiza prácticamente durante toda la jornada extraescolar, siendo el promedio de horas de exposición a la televisión de 3 horas diarias, es decir, que al mes los niños y las niñas ven en promedio por lo menos unas 90 horas de televisión.
Tabla 1. Programas preferidos de los niños y las niñas

\begin{tabular}{|c|c|c|c|}
\hline $\begin{array}{c}\text { Nombre del } \\
\text { programa } \\
\text { escogido }\end{array}$ & $\begin{array}{c}\text { Horario de } \\
\text { emisión }\end{array}$ & $\begin{array}{c}\text { Canal de } \\
\text { emisión }\end{array}$ & $\begin{array}{c}\text { Frecuencia } \\
\text { de emisión }\end{array}$ \\
\hline Hi 5 & Día & $\begin{array}{c}\text { Señal } \\
\text { Colombia }\end{array}$ & Diario \\
\hline $\begin{array}{c}\text { Los padrinos } \\
\text { mágicos }\end{array}$ & $\begin{array}{c}\text { Medio día y } \\
\text { noche }\end{array}$ & $\begin{array}{c}\text { Discovery Kids, } \\
\text { Jetix, Disney } \\
\text { Channel }\end{array}$ & Diario \\
\hline Power Rangers & Tarde & Jetix & Diario \\
\hline $\begin{array}{c}\text { Aventuras de } \\
\text { Brandy y el } \\
\text { señor Bigotes }\end{array}$ & $\begin{array}{c}\text { Día y tarde } \\
\text { Disney } \\
\text { Channel }\end{array}$ & Diario \\
\hline Dragon Ball Z & $\begin{array}{c}\text { Se emite } \\
\text { varias veces } \\
\text { al día, en la } \\
\text { mañana o en } \\
\text { la tarde. }\end{array}$ & $\begin{array}{c}\text { Network y City } \\
\text { TV }\end{array}$ & Diario \\
\hline Rebelde & Tarde & Canal 4 & Diario \\
\hline
\end{tabular}

Los resultados, además, mostraron que de los 8 niños, cuatro ven televisión a solas, dos con sus hermanos, generalmente mayores, y dos realizan esta actividad con alguno de sus padres. Estos datos indican que tanto las niñas como los niños tienden a ver la televisión sin la compañía de un adulto responsable.

Los datos también mostraron que los niños y las niñas que ven televisión en compañía de adultos o de personas mayores que ellos, incluyen dentro de los programas que ven novelas, realitys y seriados dirigidos a la audiencia familiar que se emiten a través de los canales nacionales. No obstante, cuando se les interroga sobre sus programas preferidos hacen referencia a los que corresponden a la audiencia infantil.

Ver tabla No.2 en la siguiente página 
Tabla 2. Horario en que los niños y las niñas ven TV y persona que acompaña la actividad

\begin{tabular}{|c|c|c|c|}
\hline \multicolumn{2}{|c|}{ Horario en el que ven TV } & \multicolumn{2}{|c|}{ Con quién ven TV } \\
\hline Niñas & Niños & Niñas & Niños \\
\hline 6pm - 12pm & 3pm - 7pm & $\begin{array}{c}\text { Sola o con la } \\
\text { niñera }\end{array}$ & Solo \\
\hline 6pm - 8pm & $5 p m-8 p m$ & Sola & $\begin{array}{l}\text { Hermano de } \\
14 \text { años }\end{array}$ \\
\hline 6pm - 8pm & 4pm - 8:30pm & $\begin{array}{l}\text { Hermano de } \\
10 \text { años }\end{array}$ & Papá o mamá \\
\hline $4 p m-8 p m$ & $5 p m-7 p m$ & $\begin{array}{l}\text { Dos hermanos } \\
\text { y la mamá, } \\
\text { se rotan los } \\
\text { programas }\end{array}$ & $\begin{array}{c}\text { Abuela ve al } \\
\text { mismo tiempo } \\
\text { TV en el otro } \\
\text { piso }\end{array}$ \\
\hline
\end{tabular}

Cabe señalar que durante el taller, los niños y las niñas mencionaron que los programas que más ven sus mamás son las telenovelas y los "programas de chismes" y farándula, mientras que los papás prefieren los noticieros y los programas deportivos. Estas percepciones son consistentes con los de otros estudios acerca de hábitos de consumo según el sexo del televidente.

\section{Características de los personajes preferidos}

El análisis de los dibujos sobre los personajes preferidos mostró que tanto los de las niñas como los de los niños hacen referencia a personajes masculinos y femeninos cuyas edades oscilan entre los 4 y los 25 años, siendo elegidos con mayor frecuencia los personajes adolescentes y adultos jóvenes.

Con respecto a las ocupaciones de los personajes escogidos con mayor frecuencia por los niños, se encontró que se desempeñan como guerreros o héroes, mientras que las niñas escogieron personajes

\section{Aprendizajes}

con ocupaciones diversas, por ejemplo: presentadores, estudiantes adolescentes, aquellos que tienen nexos familiares (padrino y madrina) o que se dedican a hacer deporte o no tienen una ocupación definida.

En cuanto al tema de los programas en los que aparecen los personajes preferidos se encontraron diferencias en función del sexo. Las niñas se refieren a programas de humor, donde se describen las relaciones entre las personas o los personajes, programas educativos o donde se les enseña a realizar actividades manuales. A diferencia de los niños, ellas no mencionan programas con contenido de violencia explícita. Como se observa en la taba 3, entre las características que resaltan de sus personajes preferidos incluyen el humor, la apariencia física, la ayuda hacia los demás, la colaboración y la bondad.

Los niños, por su parte, prefieren personajes de programas cuya trama central exalta los poderes de un héroe, que generalmente tiene la responsabilidad de salvar el mundo de personajes malvados. De acuerdo con los niños las características de sus personajes preferidos incluyen el poder sobre los demás, la defensa de los débiles, el castigo a los malos, las habilidades físicas y la disposición a enfrentar el peligro que implica salvar el mundo.

Tabla 3. Características del personaje preferido según sexo

\begin{tabular}{|c|c|}
\hline Niñas & Niños \\
\hline Su actitud & Poderes \\
\hline Es chistoso & Vence a los malos \\
\hline Acciones buenas & Transformarse \\
\hline Nos hacen reír & Pelean \\
\hline Bonita & Rescatan al mundo \\
\hline Ayudar a los amigos & Castigar a los malos \\
\hline Juega y canta & \\
\hline
\end{tabular}




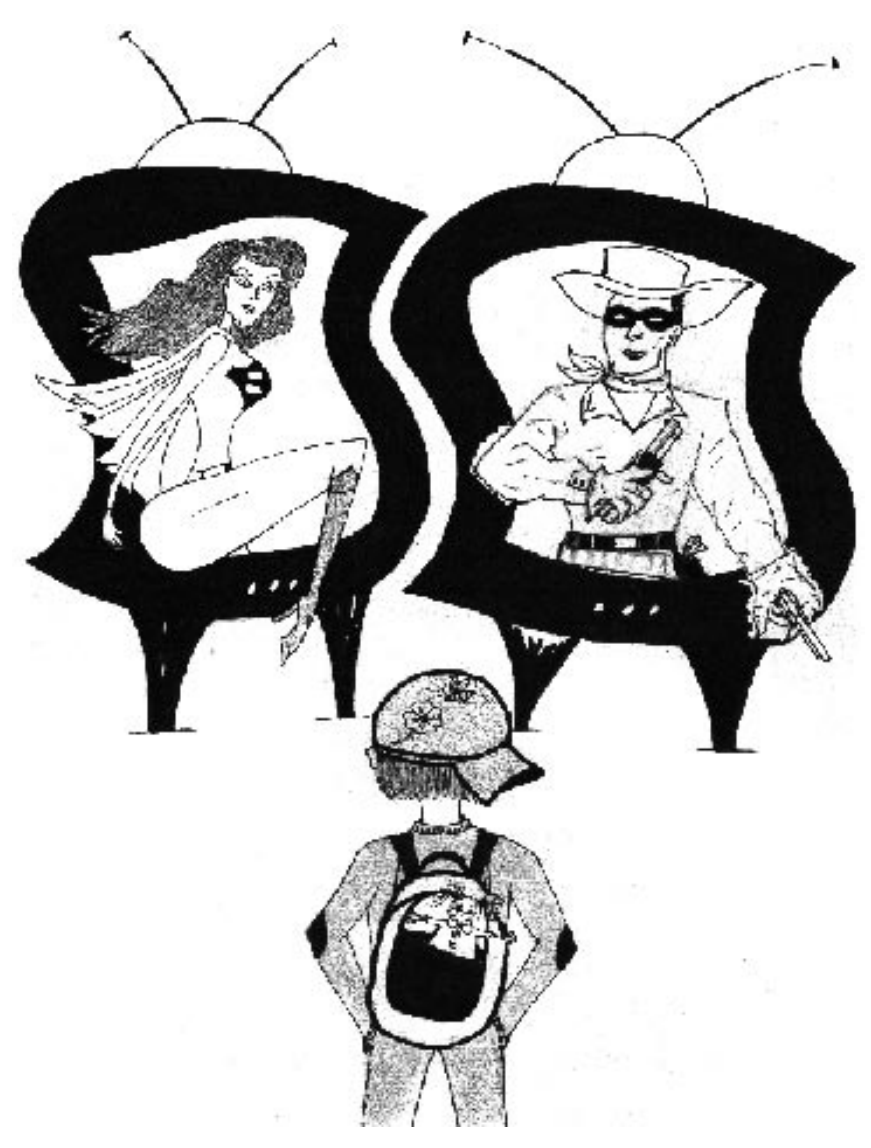

Un hecho interesante es que durante el taller, los niños descalificaron algunos de los programas que ven las niñas porque los consideran infantiles, es decir, dirigidos a "niños pequeños". Sin embargo, llamaba la atención que mientras las niñas describían sus dibujos, los niños intervenían con frecuencia para corregir o complementar la exposición de sus compañeras, revelando un conocimiento profundo de la trama y de los personajes. Cuando se les preguntó si ellos veían estos programas, respondieron afirmativamente, aunque aclararon que no los consideran adecuados porque son "tontos" o porque son "para niñas".

Por otro lado, en los dibujos elaborados durante el taller sobre los personajes preferidos también se observan diferencias de forma entre los niños y las niñas. Las niñas tienden a elaborar dibujos ubicados preferentemente en la parte izquierda de la hoja, dejando más de la mitad del papel en blanco (se les proporcionó para la actividad medio pliego de papel periódico), en los que se destacan figuras femeninas y detalles relacionados con el vestuario, el peinado y la naturaleza. En contraste los niños realizan sus dibujos en el centro de la hoja, los cuales la mayoría ocupan toda la hoja y en los que se observan principalmente personajes masculinos, héroes con poderes, con muy pocos detalles sobre el contexto o la naturaleza.

Análisis de los programas preferidos desde la perspectiva de género

Consistentemente con los hallazgos de otras investigaciones realizadas desde la perspectiva de los adultos, los resultados del estudio revelan que la interpretación que realizan las niñas y los niños de los mensajes de género que trasmiten sus programas preferidos da cuenta de fuertes estereotipos de género y actitudes sexistas. Esto se evidencia cuando analizan sus personajes preferidos en función de los roles que desempeñan, el tipo de decisiones que toma, la manera como expresan sus emociones y el grado de participación en el manejo del poder.

Los datos que se presentan en la tabla 4 indican que los niños prácticamente no incluyen personajes femeninos, mientras las niñas incluyen ambos tipos de personajes al responder las preguntas. En cuanto a los roles que desempeñan, tanto niños como niñas relacionan 
los personajes masculinos con roles tradicionales de recreación (ver TV y divertirse), si bien se observa que los niños también incluyen, aunque con menor frecuencia, roles no tradicionales como las actividades domésticas. También se observa que niños y niñas asocian los personajes femeninos con roles tradicionales de tipo doméstico (preparar la comida, hacer el aseo, supervisar las tareas). En comparación con los niños, las niñas asocian los personajes femeninos con mayor diversidad de roles no tradicionales como reparar el carro, hacer deporte, divertirse.

Tabla 4. Roles que los niños atribuyen a los personajes de sus programas preferidos

\begin{tabular}{|l|l|}
\hline \multicolumn{1}{|c|}{ Niños } & \multicolumn{1}{c|}{ Niñas } \\
\hline $\begin{array}{l}\text { Los personajes masculinos } \\
\text { son los encargados de: }\end{array}$ & $\begin{array}{l}\text { Los personajes masculinos } \\
\text { son los encargados de: }\end{array}$ \\
\hline Ver TV & Ver TV \\
\hline Divertirse & Divertirse \\
\hline Liderar & Reparar \\
\hline Dar dinero & $\begin{array}{l}\text { Reparar el carro } \\
\text { Conquistar } \\
\text { Cuidar niños }\end{array}$ \\
\hline $\begin{array}{l}\text { Con menor frecuencia, se } \\
\text { encargan de: }\end{array}$ & \\
\hline Preparar comida & \\
\hline Cuidar niños & \\
\hline Realizar aseo & \\
\hline Trabajar & \\
\hline Recibir dinero & \\
\hline Comprar & \\
\hline Revisar tareas & \\
\hline Aseo personal & \\
\hline
\end{tabular}

\begin{tabular}{|l|l|}
\hline $\begin{array}{l}\text { Los personajes femeninos se } \\
\text { encargan de: }\end{array}$ & $\begin{array}{l}\text { Los personajes femeninos se } \\
\text { encargan de: }\end{array}$ \\
\hline Preparar comida & Comprar \\
\hline Revisar tareas & Preparar la comida \\
\hline Realizar aseo & Revisar tareas \\
\hline Dar dinero & Realizar aseo personal \\
\hline Reparar & Realizar aseo \\
\hline & Dar dinero \\
\hline & Liderar \\
\hline & Llevar al hospital \\
\hline & Conquistar \\
\hline & Cuidar los niños \\
\hline & Con mucha \\
menor frecuencia:
\end{tabular}

Cuando se analiza el tipo de decisiones que, desde la perspectiva de los niños y las niñas, toman los personajes de sus programas preferidos se encuentra que ambos afirman que los personajes masculinos se encargan de definir lo concerniente a los castigos y lo que se puede o no hacer. Tanto niños como niñas asocian los personajes femeninos con decisiones como organizar la casa, las tareas que se debe realizar y la manera de solucionar los problemas. En la tabla 5 se aprecia que tanto niñas como niños perciben que los personajes de su propio sexo toman mayor número y diversidad de decisiones. 
Tabla 5. Tipo de decisiones que toman personajes masculinos y femeninos

\begin{tabular}{|l|l|}
\hline \multicolumn{1}{|c|}{ Niños } & \multicolumn{1}{|c|}{ Niñas } \\
\hline $\begin{array}{l}\text { Los personajes masculinos } \\
\text { deciden sobre: }\end{array}$ & $\begin{array}{l}\text { Los personajes masculinos } \\
\text { deciden sobre: }\end{array}$ \\
\hline Los castigos & Los castigos \\
\hline $\begin{array}{l}\text { Lo que se puede o } \\
\text { no hacer }\end{array}$ & $\begin{array}{l}\text { Lo que se puede hacer } \\
\text { o no hacer }\end{array}$ \\
\hline Cómo organizar la casa & $\begin{array}{l}\text { El carro que se } \\
\text { debe comprar }\end{array}$ \\
\hline $\begin{array}{l}\text { La manera de solucionar los } \\
\text { problemas }\end{array}$ & Ir al médico \\
\hline $\begin{array}{l}\text { Con menor frecuencia } \\
\text { deciden sobre: }\end{array}$ & Cuándo comprar casa \\
\hline Las cosas importantes & problemas \\
\hline Cómo gastar el dinero & Las actividades escolares \\
\hline Qué tareas se realizan & \\
\hline Las actividades escolares & \\
\hline Carro que comprar & Lué tareas se realizan \\
\hline Tipo de carro & Las cosas importantes \\
\hline Cuándo comprar casa & Cómo gastar el dinero \\
\hline Cómo organizar la casa & \\
\hline Qué tareas se realizan & \\
\hline $\begin{array}{l}\text { La manera de solucionar los } \\
\text { deninos }\end{array}$ \\
\hline
\end{tabular}

\begin{tabular}{|l|l|}
\hline & $\begin{array}{l}\text { El carro que se } \\
\text { debe comprar }\end{array}$ \\
\hline & $\begin{array}{l}\text { Lo que se puede o no se } \\
\text { puede hacer }\end{array}$ \\
\hline & Ir al médico \\
\hline & Cuándo comprar casa \\
\hline
\end{tabular}

Al examinar las respuestas de los participantes en el taller, a la pregunta sobre las emociones que expresan con mayor frecuencia los personajes de sus programas preferidos, se encuentra que tanto niños como niñas asocian los personajes masculinos con preocupación. Los niños, adicionalmente, relacionan los personajes masculinos con manifestaciones como la alegría, el amor y la rabia. Las niñas, por su parte, asocian a los personajes femeninos con emociones como la alegría, la tranquilidad y el amor, al igual que con la rabia y el dolor. En la tabla 6 se observa que los niños no identificaron expresiones emocionales por parte de los personajes femeninos.

\section{Ver tabla No.6 en la siguiente página}

Frente a la pregunta sobre quién es el personaje que ocupa el primer lugar en distintas situaciones, se encontró que tanto niñas como niños coinciden en mencionar con mayor frecuencia que los personajes masculinos de sus programas preferidos se distinguen porque pelean más. Las niñas perciben que los personajes masculinos se destacan como líderes negativos, no sólo son los que más pelean, también dan mal ejemplo, tratan mal a los demás y se portan mal. En su opinión, también ocupan el primer lugar en pedir perdón. Tanto niños como niñas perciben que el personaje de su propio sexo se distingue por ser el más querido, admirado, colaborador, afectuoso, dar buen ejemplo y por su buen comportamiento. Al igual que 
en el análisis de las expresiones emocionales, en este apartado de la guía de análisis los niños no tuvieron en cuenta los personajes femeninos.

Tabla 6. Expresiones emocionales de los personajes femeninos y masculinos

\begin{tabular}{|l|l|}
\hline \multicolumn{1}{|c|}{ Niños } & \multicolumn{1}{c|}{ Niñas } \\
\hline $\begin{array}{l}\text { Los personajes masculinos } \\
\text { se asocian con: }\end{array}$ & $\begin{array}{l}\text { Los personajes masculinos } \\
\text { se asocian con: }\end{array}$ \\
\hline Alegría & Preocupación \\
\hline Amor & tristeza \\
\hline Rabia & \\
\hline Con menor frecuencia: & \\
\hline Tranquilidad & \\
\hline Preocupación & \\
\hline Dolor & \\
\hline $\begin{array}{l}\text { Los niños no identificaron } \\
\text { emociones asociadas con } \\
\text { los personajes femeninos }\end{array}$ & Los personajes femeninos se \\
\hline & Alegría \\
\hline & Tranquilidad \\
\hline & Tristeza \\
\hline & Rabia \\
\hline & Dolor \\
\hline & Amor \\
\hline
\end{tabular}

Tabla 7. Características y comportamientos por los cuales se destacan los personajes femeninos y masculinos

\begin{tabular}{|l|l|}
\hline \multicolumn{1}{|c|}{ Niños } & \multicolumn{1}{c|}{ Niñas } \\
\hline $\begin{array}{l}\text { Los personajes masculinos } \\
\text { ocupan el primer lugar por: }\end{array}$ & $\begin{array}{l}\text { Los personajes masculinos } \\
\text { ocupan el primer lugar por: }\end{array}$ \\
\hline Ser quien más pelea & Dar buen ejemplo \\
\hline
\end{tabular}

\section{Aprendizajes}

\begin{tabular}{|c|c|}
\hline Ser el más querido & $\begin{array}{l}\text { Ser quien manda a } \\
\text { los demás }\end{array}$ \\
\hline Ser el más admirado & Ser quien más pelea \\
\hline Se el más colaborador & $\begin{array}{l}\text { Ser quien trata mal a } \\
\text { los demás }\end{array}$ \\
\hline Dar buen ejemplo & Ser quien se porta mal \\
\hline Ser quien pide perdón & Ser quien pide perdón \\
\hline \multicolumn{2}{|l|}{$\begin{array}{l}\text { Ser quien manda a } \\
\text { los demás }\end{array}$} \\
\hline \multicolumn{2}{|l|}{ Ser el más afectuoso } \\
\hline \multicolumn{2}{|l|}{ Ser quien se porta bien } \\
\hline \multirow{15}{*}{$\begin{array}{l}\text { No identifican personajes } \\
\text { femeninos que ocupen } \\
\text { el primer lugar en algún } \\
\text { aspecto. }\end{array}$} & $\begin{array}{l}\text { Los personajes femeninos } \\
\text { ocupan el primer lugar } \\
\text { por: }\end{array}$ \\
\hline & Ser el más querido \\
\hline & Ser el más admirado \\
\hline & Ser el más colaborador \\
\hline & Dar buen ejemplo \\
\hline & Evitar el conflicto \\
\hline & Ser quien se porta mal \\
\hline & Ser quien se porta bien \\
\hline & $\begin{array}{l}\text { Mencionaron con menor } \\
\text { frecuencia que el personaje } \\
\text { femenino ocupa el primer } \\
\text { lugar por: }\end{array}$ \\
\hline & Ser el más exitoso \\
\hline & Dar mal ejemplo \\
\hline & Ser quien pide perdón \\
\hline & $\begin{array}{l}\text { Ser quien manda a } \\
\text { los demás }\end{array}$ \\
\hline & Ser quien más pelea \\
\hline & Ser el más afectuoso \\
\hline
\end{tabular}




\begin{tabular}{|l|l|}
\hline & $\begin{array}{l}\text { Ser quien trata mal a los } \\
\text { demás }\end{array}$ \\
\hline & Ser el que se porta bien \\
\hline & Ser el que se porta mal \\
\hline
\end{tabular}

\section{Discusión}

Este estudio ha tenido como propósito fundamental establecer el conocimiento acerca del género que están adquiriendo los niños y las niñas a través de la televisión. Para cumplir con esta finalidad se invitó a 4 niños y 4 niñas de segundo grado de primaria a un taller en el que proporcionaron información sobre sus hábitos de consumo televisivo, al igual que sobre las características de sus programas y personajes preferidos. En el taller también se involucró a las niñas y los niños en el proceso de indagación sobre el contenido de sus programas preferidos, teniendo en cuenta como categorías para el análisis del género: a) las actividades que se representan en los mensajes y que ilustran la división sexual del trabajo en términos de roles productivos, reproductivos y de participación comunitaria; b) las características emocionales que los participantes perciben de los personajes del programa elegido; c) las relaciones de poder que se representan y que se expresan a través de la posición que ocupan los personajes en la trama y la manera como resuelven los conflictos, toman decisiones y participan en las actividades.

Con respecto a los hábitos de consumo, los resultados muestran que a pesar de las nuevas tecnologías, la televisión continúa siendo el principal medio de recreación de los niños y las niñas consultados, al menos durante la jornada extraescolar en la que, prácticamente, se dedican a ver televisión. Esta excesiva exposición a la televisión no sería tan preocupante si los niños y las niñas hubieran reportado que la actividad la realizan en compañía de las personas adultas que se encargan de su cuidado. Esto dado que la evidencia disponible indica que los padres o acudientes pueden modular el efecto de los contenidos acompañando a sus hijos mientras ven televisión y propiciando un ambiente en el que se pueda hablar acerca del contenido de los programas (Strasburger, 1995).

Los datos de este estudio señalan que la sugerencia de los organismos interesados en el tema, para que los niños y las niñas vean televisión en compañía de "adultos responsables" no está siendo considerada. Los relatos de los participantes también indican que, contrariamente a lo que proponen investigadores como Verma y Larson (2002), la supervisión que tienen del uso de la televisión es limitada, probablemente porque las personas con quienes conviven no disponen de alternativas para cumplir con esta práctica de manera efectiva.

Cabe señalar que no es la cantidad de televisión que se ve lo que más influye sobre las actitudes, expectativas y comportamientos del televidente (Vargas Trujillo, Barrera, Burgos y Daza, 2003; Ward, 2002; Ward \& Rivadeneyra, 1999, entre otros). No obstante, conviene tener en consideración que si bien los niños y las niñas son selectivos, en tanto que a la hora de elegir sus programas favoritos optan por los que son indicados para su edad, cuando ven programas en compañía de jóvenes o adultos tienden a ver programas como novelas y seriados que no son aptos para la audiencia infantil. Estos datos sugieren que las personas que acompañan a los niños y a las niñas a ver televisión no priorizan la programación infantil y las necesidades de los más pequeños de la familia. 
Los hallazgos de diversos estudios previos plantean que la exposición frecuente a novelas y seriados puede tener un efecto importante en la construcción de modelos, reglas y estándares de feminidad y masculinidad (Bem, 1981a; Lytton \& Romney, 1991; Meth, 1990 citado por Mahalik, 2005). En Colombia, el estudio de Rojas (2007) sobre identidad de género y consumo cultural televisivo en jóvenes, mostró que tanto las mujeres que escogen como primera opción las novelas como los hombres que eligen como primera opción los programas deportivos, presentan altos puntajes en actitudes sexistas. Por otra parte, las personas que tienden a optar por programas como los informativos o investigativos presentan puntajes más bajos en las escalas que miden dichas cogniciones.

Como ya se ha señalado, el consumo mediático no es un proceso unidireccional, en la interpretación de los mensajes de género que trasmite la televisión es indudable el papel que pueden tener otros agentes de socialización como padres, profesores y pares. A través de ellos, los niños y las niñas también conocen diversos elementos para diferenciar a hombres de mujeres. Sin embargo, los resultados del presente estudio con relación a los programas preferidos por los padres y las madres, según los niños, permiten inferir que las personas adultas con quienes conviven presentan altos niveles de sexismo y, por lo tanto, en la cotidianidad se pueden estar reforzando aquellas reglas y estándares que conducen

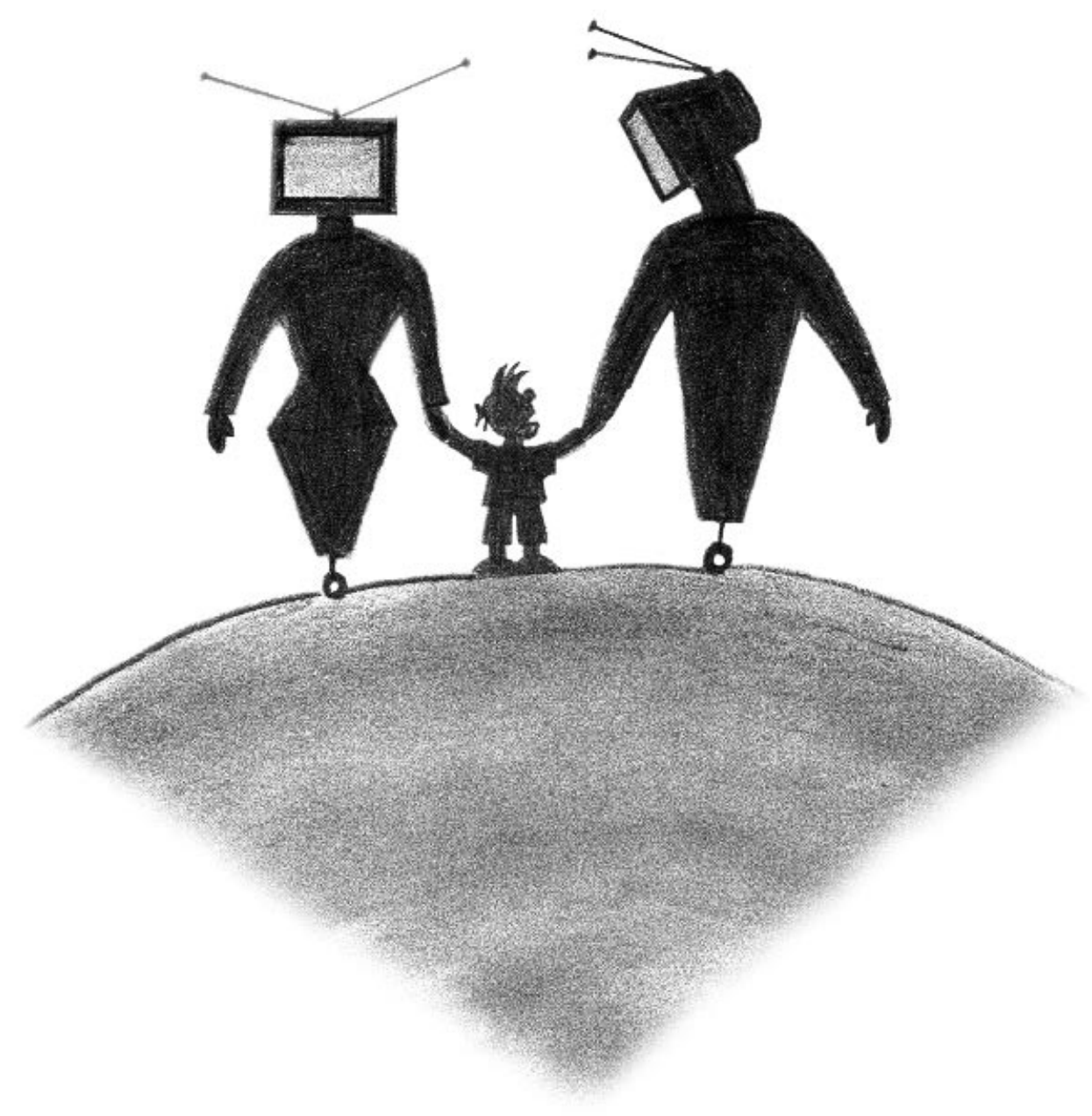


a tratos discriminatorios entre los grupos de iguales. Estos datos se complementan con los hallazgos de este estudio sobre la percepción que tienen los participantes de los personajes de sus programas preferidos. En el taller se logró establecer que los programas con protagonistas pre-adolescentes y adultos jóvenes son los que más interesan a los niños y las niñas de 8 a 9 años de edad. Por lo tanto, las características de género que perciben en estos personajes son relevantes, en tanto que constituyen modelos de identificación para la población infantil.

En este sentido, conviene considerar que las interpretaciones de género que realizaron los niños y las niñas de sus programas preferidos, dan cuenta de fuertes estereotipos de género y actitudes sexistas. Esto se evidencia cuando analizan sus personajes preferidos en función de los roles que desempeñan, la manera como expresan sus emociones, el tipo de decisiones que toman y el grado de participación en el manejo del poder.

En cuanto a los roles que desempeñan, se encontró que las niñas consultadas asocian a los personajes femeninos con actividades de cuidado y reproductivas, en las que priman las relaciones sociales, la ayuda a los demás y la colaboración en situaciones cotidianas. Por su parte, los niños atribuyen a los personajes masculinos actividades cuyo desempeño exige poderes extraordinarios, son héroes, que tienen la responsabilidad de salvar el mundo, la obligación de defender a los más débiles y el poder de castigar a los malos. Esta apreciación que hacen los participantes en el taller de los personajes de sus programas preferidos, apoya los resultados de diversos estudios acerca de que en las producciones televisivas persiste la elección de roles femeninos y masculinos fuertemente estereotipados, opuestos y complementarios (Charles, 2002; Bengoechea, 2006; Göłz, 2008; Hains, 2008).
La percepción sexista que tienen los niños y las niñas de los personajes que aparecen en sus programas preferidos también se hizo evidente en la valoración que hacen de sus expresiones emocionales y de la manera como ejercen el poder. Este tipo de representaciones impide que los niños y las niñas tengan acceso a modelos de identificación diversos en los que la masculinidad no esté asociada, exclusivamente, con la violencia y el ejercicio del poder, y la feminidad con la belleza física, la expresión emocional y las relaciones sociales.

Esta situación es particularmente alarmante si se tiene en cuenta que aunque la oferta de programas con alto contenido sexista es la de mayor presencia en la televisión, este medio de comunicación promueve poco o nada los aprendizajes para que los niños y las niñas se conviertan en receptores activos de los medios (Niño, 2004). De otro lado, conviene señalar que si bien los realizadores, productores y guionistas de televisión, especialmente de América Latina, pueden estar dispuestos a realizar una televisión diferente (CILA, 2007), no cuentan con orientación especializada que les permita tener criterios mínimos para realizar programas con perspectiva de género.

En este sentido, vale la pena considerar que no es posible pensar en promover a través de la televisión la equidad de género, si no se incluyen a los niños y las niñas en el proceso de planificación de las iniciativas. Concretamente, pensar en realizar un análisis de aquello que ofrece la televisión sin contar con la interpretación de los niños y las niñas o en definir proyectos educativos en género sin dar herramientas a la audiencia infantil para analizar medios con esta perspectiva, no es coherente con los nuevos modelos de educación en comunicación que incluyen la participación como elemento sustancial de las propuestas. 


\section{Conclusiones}

De acuerdo con los hallazgos de otras investigaciones realizadas desde la perspectiva de los adultos, los resultados del estudio revelan que la interpretación que realizan las niñas y los niños de los mensajes de género que trasmiten sus programas preferidos da cuenta de fuertes estereotipos de género y actitudes sexistas. Esto se evidencia cuando analizan sus personajes preferidos en función de los roles que desempeñan, los atributos de personalidad que los caracterizan, la manera como expresan sus emociones, el tipo de decisiones que toman y el grado de participación en el manejo del poder.

La interpretación que, desde la perspectiva de género, hicieron las niñas y los niños de sus programas preferidos y de los personajes que en ellos se representan, muestra el conocimiento acerca del género que están adquiriendo a través de la televisión:

1. Los mundos masculino y femenino, representados en los personajes de los programas favoritos de los niños y las niñas, parecen no tener puntos de encuentro y ser diametralmente opuestos. El mundo masculino es percibido por los niños como caracterizado por la violencia, el poder, la lucha y el castigo contra la maldad. Desde la perspectiva de las niñas, el mundo femenino está caracterizado por el buen comportamiento, la imagen física y social que se proyecte y el buen humor. Estas diferencias que se representan en la televisión entre ambos mundos restringen las posibilidades que tienen los niños y las niñas para desarrollar todas sus potencialidades físicas, afectivas, sociales, intelectuales y trascendentes y definir proyectos de vida sin las limitaciones que imponen las normas y expectativas de género.
2. Las diferencias encontradas en la manera como los niños interpretan los roles que asumen los personajes masculinos y femeninos, reafirman y reproducen las diferencias aún existentes en el campo del trabajo en el mundo adulto. Los niños y las niñas están construyendo la noción de que los personajes masculinos son quienes se divierten, poseen poderes extraordinarios, asumen el liderazgo y pocas veces o nunca se encargan de actividades reproductivas o domésticas. Los personajes femeninos, en contraste, asumen preferentemente los roles tradicionales reproductivos como las actividades domésticas y familiares. Afortunadamente, los datos sugieren que tanto niños como niñas comienzan a vislumbrar la posibilidad de que los personajes adopten algunos roles no tradicionales, especialmente cuando se refieren al personaje de su mismo sexo.

3. Desde la perspectiva de los niños y las niñas, las relaciones de poder que se establecen entre los personajes, la manera como resuelven los conflictos, toman decisiones y participan en las actividades, están claramente diferenciadas para ambos sexos. Los personajes masculinos son percibidos por niños y niñas como poderosos, castigadores y con la responsabilidad de defender a los débiles y salvar el mundo de los peligros que acechan. Es decir, la responsabilidad del universo recae sobre ellos, mientras que las decisiones cotidianas (cómo organizar la casa, qué tareas realizar o cómo solucionar los problemas) están a cargo de ellas. Estas percepciones plantean un mundo donde los hombres no se ocupan de lo cotidiano, lo doméstico y las mujeres no se encargan de lo "importante", lo público. Estas interpretaciones contrastan con el conocimiento que existe hoy sobre los derechos y posibilidades de ambos sexos para actuar de manera diversa en el mundo. 


\section{Megicariomes}

4. Otra diferencia notable en las interpretaciones de los niños y las niñas se observa cuando se analiza el tipo de liderazgo que le atribuyen a los personajes de sus programas preferidos. Aunque los personajes masculinos son percibidos tanto por los niños como por las niñas como líderes negativos, son las niñas quienes tienen una percepción más negativa de estos personajes (son quienes más pelean, dan mal ejemplo, se portan mal). En contraste, los personajes femeninos son percibidos por las niñas como líderes sociales (son las que dan buen ejemplo, las más queridas, las más admiradas). Llama la atención que los niños no identifican personajes femeninos que se destaquen en algún aspecto dentro de su programa preferido. Tampoco lograron reconocer las emociones que se asocian con los personajes femeninos. Estos son dos datos que vale la pena someter a examen en investigaciones futuras.

5. Los resultados sobre los conocimientos que los niños y las niñas están adquiriendo sobre las relaciones de género a través de sus programas favoritos, reafirman la necesidad de trabajar tanto en la formación
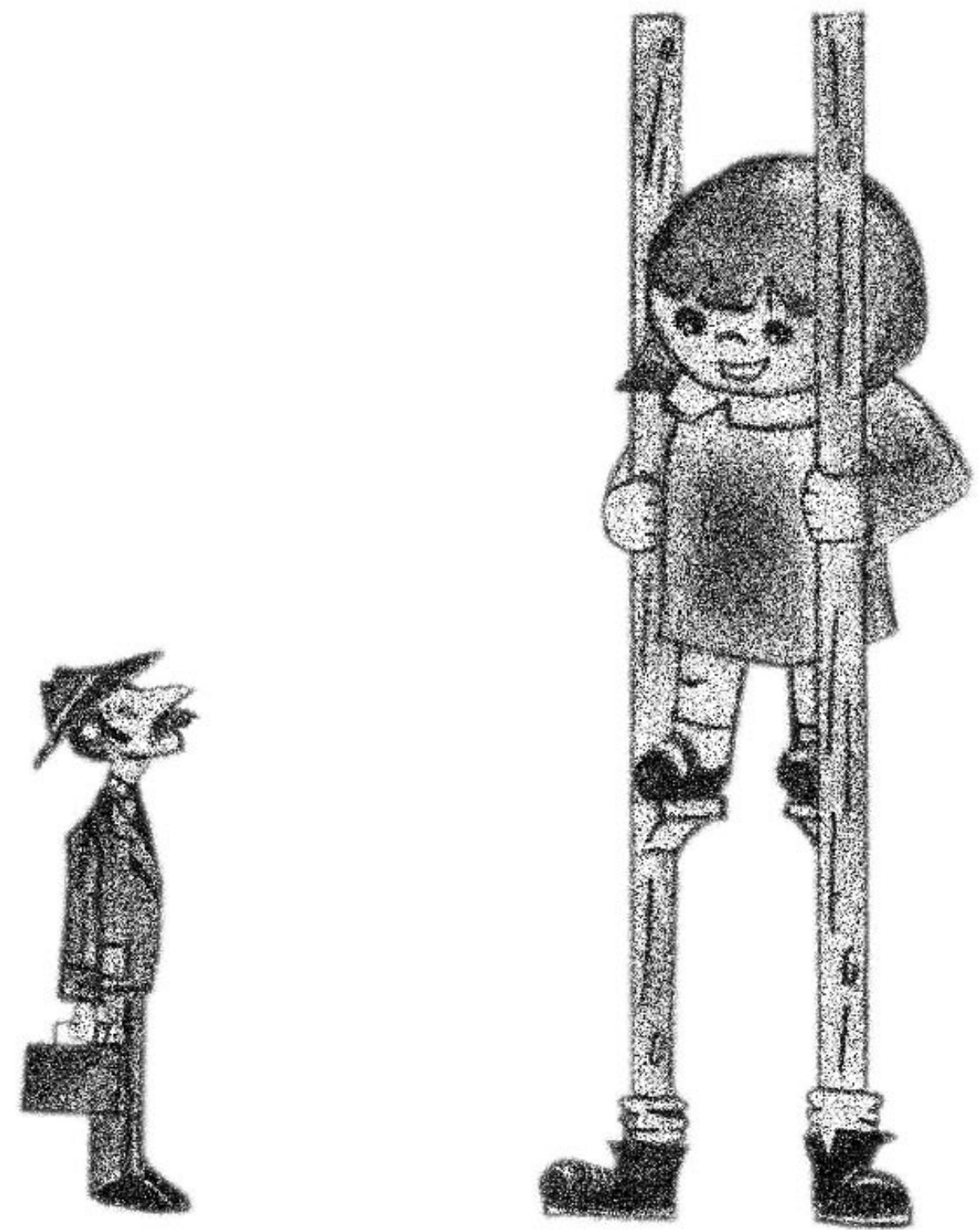


\section{Aprendizajes}

de competencias críticas con perspectiva de género en la población infantil y adulta, acerca de lo que ven en televisión, como en la producción de una más amplia oferta televisiva que permita a niños y a niñas vislumbrar la gran diversidad de roles, características, actividades y formas de relación existentes, las cuales no son patrimonio de uno u otro sexo.

\section{Bibliografía}

AUFENANGER, S., "Stimuli, not set answers", en: Televizion $18,2005$.

BEGOECHEA, M., DÍAZ-AGUADO, M., FALCON, L., LÓPEZ, P., y PÉREZ, Ángeles, Infancia, televisión y género. Guía para la elaboración de contenidos no sexistas en programas infantiles de televisión, Barcelona, Secretaría General de Políticas de Igualdad, Instituto de la Mujer, RTVE Instituto, 2006.

BEM, S., "Gender schema theory: a cognitive account of sex typing", en: Psychological Review, 88, 4, 1981.

CAZÉS, D. , La perspectiva de género: guía para diseñar, poner en marcha, dar seguimiento y evaluar proyectos de investigación y acciones públicas y civiles, México, CONAPO-PRONAM, 1998.

CHARLES, M., "El Género en la televisión", en: Revista Signo y Pensamiento, Bogotá, Pontificia Universidad Javeriana, 2002

ClLA, Entrevista con Esteban Geeofroy Pita, realizada por la Iniciativa de la Comunicación, durante el evento Televisión de Calidad 2007, VI Muestra y Conferencia Internacional, realizado el 30 de agosto al 2 de septiembre de 2007 en Bogotá, Colombia.

FOX, W., \& SANTOS, C., Análisis de contenido sexual en los programas de audiencia infantil, Bogotá, Documento CESO, Universidad de los Andes, 2002.

GÖTZ, M., "Learning in knowledge and documentary programmes", en: Televizion 18, 2005. 
GÖTZ, M., HOFMANN, O., BROSIUS, H.B., et al, "Gender in Children's Television Worldwide", en: Televizion, Girls and Boys and Television, The role of Gender, Internationales Zentralinstitut fur das Jugend und Bildungsfernsehen, 2008.

HAINS, R., "Are super girls super for girl? The negotiation of beauty ideals in girl power cartoons", en: Televizion, Girls and Boys and Television, The role of Gender, Internationales Zentralinstitut fur das Jugend und Bildungsfernsehen, 2008.

LYTTON, H., \& ROMNEY, D. M., "Parents' differential socialization of boys and girls: A meta-analysis", en: Psychological Bulletin 109, 1991.

MAHALIK, J. R., MORRAY, E., COONERTY-FEMIANO, A., LUDLOW, L. H., SLATTERY, S. M., \& SMILER, A., "Development of the Conformity to Feminine Norms Inventory", en: Sex Roles 52, 2005.

NIÑ̃, D. J., Niñez, Juventud y televisión en Colombia. Una tarea aplazada, Documento de trabajo presentado en la cumbre mundial de medios para niños, niñas y adolescentes, Río de Janeiro (Brasil), 19 al 23 de Abril de 2004.

RINCÓN, O., Medios, Infancia y Adolescencia en Colombia, Informe para la cuarta cumbre mundial de medios para niños, niñas y adolescentes, Río de Janeiro (Brasil), 19 al 23 de Abril de 2004.

ROJAS, A., Identidad de Género y Consumos Culturales Televisivos: Más allá de las Diferencias por Sexo, Bogotá, trabajo de grado para optar el título de Maestría en Psicología, Universidad de los Andes, 2007.
STRASBURGER, V., Adolescents and the media: Medical and psychological impact, Thousand Oaks, Sage Publications, 1995.

THOMPSON, T. L., \& ZERBINOS, E., "Television cartoons: Do children notice it's a boy's world?", en: Sex Roles 37, 1997.

VARGAS TRUJILLO, E., \& BARRERA, F., Influencia de los programas televisivos con contenido sexual sobre el comportamiento de los adolescentes, Informe Final presentado a la Comisión Nacional de Televisión, Bogotá, Departamento de Psicología, Universidad de Los Andes, 2003.

VARGAS, E., BARRERA, $F_{1}$, BURGOS, C., y DAZA, B., Influencia de los programas televisivos con contenido sexual sobre el comportamiento de los adolescentes, Bogotá, Documentos CESO 82, Facultad de Ciencias Sociales Universidad de los Andes, 2004.

VERMA, S., \& LARSON, R. W., "Television in Indian adolescents' lives: A member of the family", en: Journal of Youth and Adolescence, 2002.

WARD, L. M., "Does television exposure affect emerging adults' attitudes and assumptions about sexual relationships? Correlational and experimental confirmation", en: Journal of Youth and Adolescence 31, 2002.

WARD, L. M., \& RIVADENEYRA, R., "Contributions of entertainment television to adolescents' sexual attitudes and expectations: The role of viewing amount versus viewer involvement", en: J. Sex Res 36, 1999. 